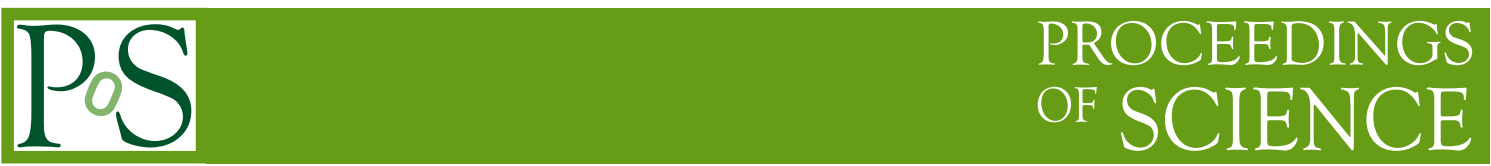

\title{
SS433, microquasars, and other transients
}

\section{Zsolt Paragi*}

Joint Institute for VLBI in Europe (JIVE), Postbus 2, 7990 AA Dwingeloo, The Netherlands

E-mail: zparagi@jive.nl

\section{René Vermeulen}

Netherlands Institute for Radio Astronomy (ASTRON), Postbus 2, 7990 AA Dwingeloo, The

Netherlands

E-mail: rvermeulen@astron.nl

\section{Ralph Spencer ${ }^{\dagger}$}

Jodrell Bank Centre for Astrophysics The University of Manchester Oxford Rd Manchester M13 9PLUK

E-mail: ralph.spencer@manchester.ac.uk

\begin{abstract}
X-ray binaries have been an important key in understanding the jet-disc symbiosis in accreting black holes on all mass scales, from stellar-mass to supermassive black holes. SS433 was the first Galactic XRB that has been extensively studied in the radio regime. The radio properties, including the highest angular resolution data can now be better understood in the framework for accretion disc state transitions that is observed in microquasars (black hole X-ray binary systems). SS433 remains unique in various ways to date, and there is still much to learn about black hole accretion phenomena. In the meantime, the electronic very long baseline (e-VLBI) developments at the European VLBI Network (EVN) has allowed us to study microquasars and other transients at milliarcsecond resolutions more flexibly than was possible before. Even more new opportunities will arise as the SKA pathfinders become operational.
\end{abstract}

Resolving the Sky - Radio Interferometry: Past, Present and Future -RTS2012

April 17-20, 2012

Manchester, UK

\footnotetext{
* Speaker.

$\dagger$ This paper is dedicated to Prof. István Fejes (1932-2011), enthusiastic researcher of SS433, who established radio astronomy (and especially VLBI) in Hungary. e-VLBI research infrastructure in Europe is supported by the European Union's Seventh Framework Programme (FP7/2007-2013) under grant agreement RI-261525 NEXPReS. The EVN is a joint facility of European, Chinese, South African and other radio astronomy institutes funded by their national research councils.
} 


\section{The first radio-jet $X$-ray binary}

A very unusual star received great attention at the end of 1970s which showed peculiar $\mathrm{H} \alpha$ emission lines: SS433, an eclipsing binary system that had counterparts in the X-ray and radio domain as well. The spectral lines showed high and variable Doppler-shifts between -30000 and $50000 \mathrm{~km} / \mathrm{s}$, and these were soon interpreted as signatures of jets precessing with a period of about 164 days [for an early review, see 1]. High resolution radio observation in 1979 with MERLIN indeed showed an elongated structure on arcsecond scale [2], and the same year, compact structure on milliarcsecond scales was reported following the first VLBI detection with a 3-station array of Effelsberg, Onsala, and the Westerbork Synthesis Radio Telescope [3]. Just in 1979, a dozen of Nature papers were published on the source, and the excitement about this fascinating object certainly played a great role in the forming of the European VLBI Network (EVN) in 1980.

And there was a lot to discover. The highest angular resolution images of the precessing radio beams allowed an accurate measurement of the source distance to $5 \pm 0.3 \mathrm{kpc}$ [e.g. 4] ${ }^{1}$ Due to the variable jet viewing angle and mildly relativistic speeds of $0.26 \mathrm{c}$, it became possible to test the Doppler-beaming paradigm for relativistic jets as well [5]. The source showed flaring episodes every $\sim 400$ days. There were two types of flares identified, one showing a delay with observing frequency, typical of synchrotron self-absorbed ejecta, the other peaked simultaneously at all frequencies -the origin of the flares were not known [6]. Besides, there was a drop in flux density observed before the flares. There were two VLBI monitoring campaigns organized in 1985 and 1987, both lasting 6 days, with 2 day separation in between the observations. These were really monster VLBI campaigns at the time, using practically the whole supplies of EVN recording tapes, weighing over a tonne. But the results were spectacular. There were a series of moving ejecta detected that originated in an elongated core, and later brightened up at an angular distance of 40-60 mas (a brightening zone) - these two regions were responsible for the two different types of flares. The 1987 campaign coincided with a major flare by chance, and a series of spectacular images were produced. The Doppler-beaming effect was confirmed both in the permanent core, as well as the in the moving ejecta, in agreement with the measured proper motion of $0.26 \mathrm{c}$ [7].

\section{The compact jets}

Multifrequency imaging with the VLBA provided new insights to the nature of the core region [8]. The brightest peaks in the approaching and receding jets have increasingly larger separation at lower frequencies. This is due to a change in synchrotron opacity: the distance of the $\tau \sim 1$ optical depth surface to the central engine is inversely proportional to the observing frequency in a self-absorbed compact jet. In active galactic nuclei (AGN) this is known as frequency dependent core-shift [9]. Indeed, the compact object in SS433 lies about midway between the eastern and western peaks of emission at $1.6 \mathrm{GHz}$ (in the centre of images in Fig.1), not in the brightest "radio core" component. This was established by fitting the kinematic model to the moving ejecta in the system. While this core-jet region has been observed most of the time in the system, there is evidence for supression of the inner jet during (at least in some of the) flares [10]; the temporal

\footnotetext{
${ }^{1}$ Note that measuring the distances of transient Galactic radio sources remains a big challenge to date; the uncertain distances and jet viewing angles still limit our ability to determine the jet Lorentz-factors accurately in black hole XRB.
} 


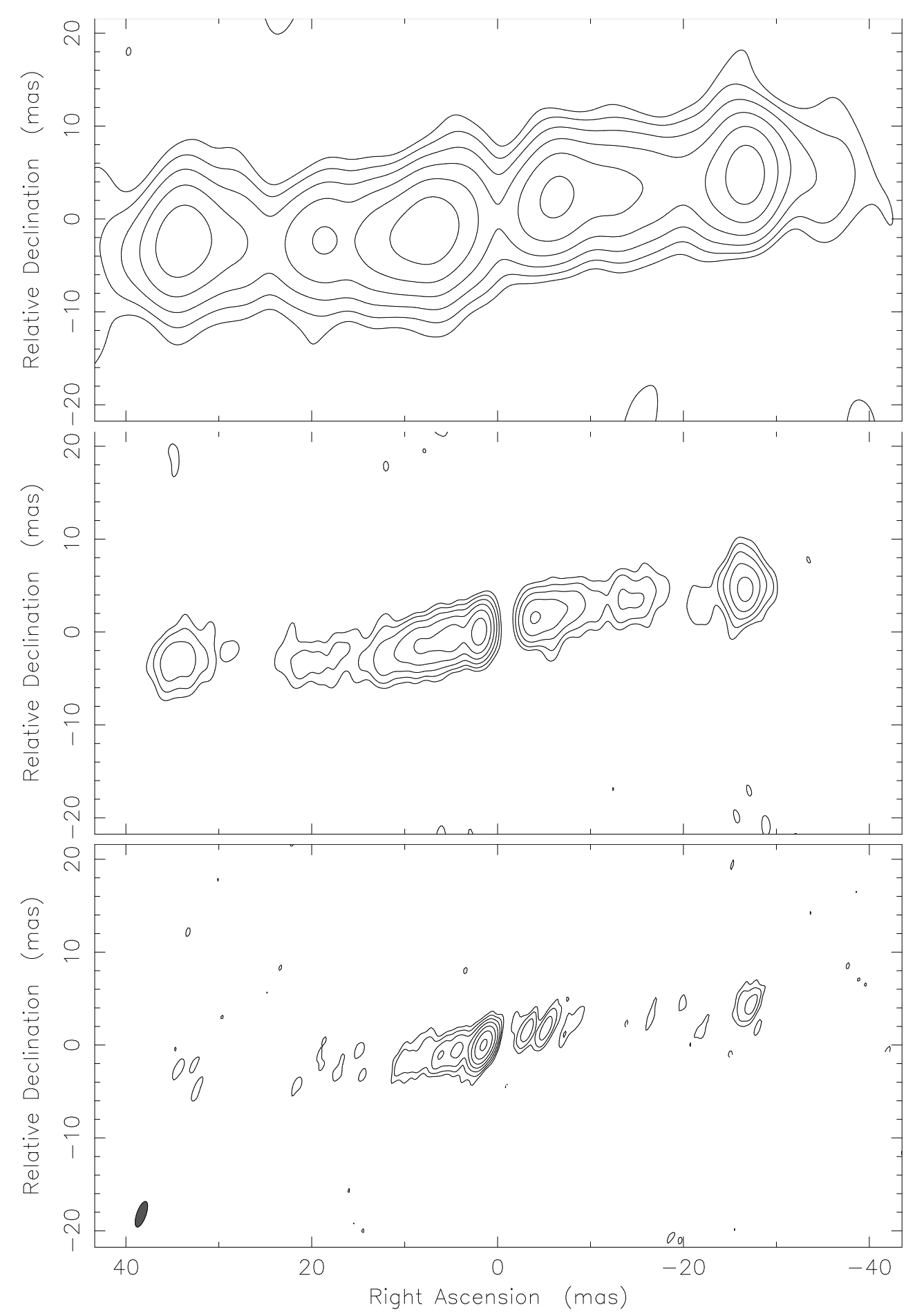

Figure 1: VLBA $1.6 \mathrm{GHz}$ (top), $5 \mathrm{GHz}$ (bottom) and $15 \mathrm{GHz}$ (bottom) maps of of SS433 on the same scale, demonstrating the multifrequency properties of the self-absorbed compact jets. The compact object is located near the centre of these maps (found by fitting the kinematic model to the ejecta). The gap between the observed "radio cores" closes at higher frequencies, this effect is known in AGN as frequency dependent core-shift. The brightness assymetry at $1.6 \mathrm{GHz}$ is consistent with Doppler-beaming; the increasing assymetry at higher frequencies is likely due to additional free-free absorption close to the central engine. 


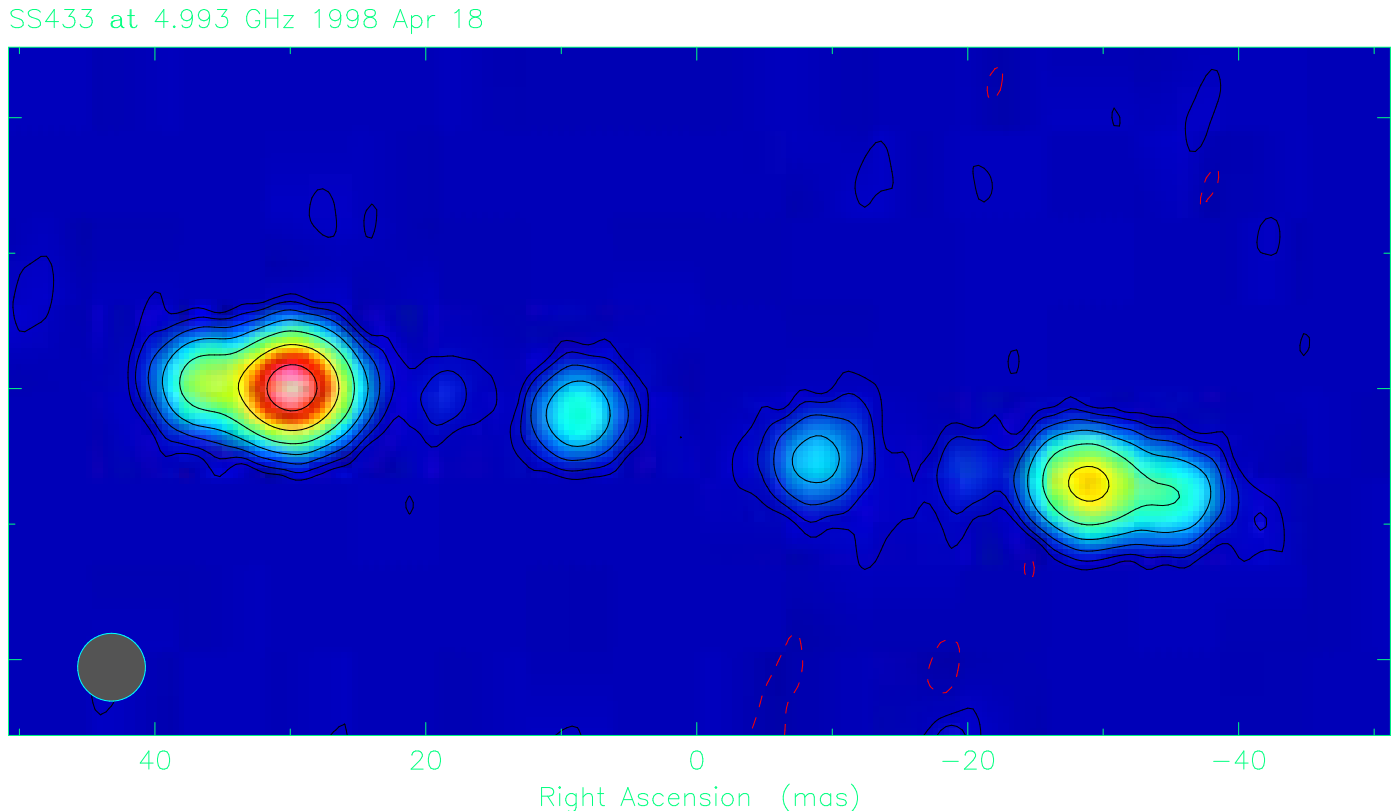

Figure 2: $5 \mathrm{GHz}$ VLBA map of SS433 during a strong flare on 18 April 1998. The core-region faded below detection, indicating that the continuous inner jets were suppressed at the beginning of the flaring event. Quenching of radio emission from the jets is now frequenctly observed in microquasars during accretion state transitions, when they enter into a soft-state, where the emission is dominated by the accretion disc. In SS433 there are no such accretion state changes observed, simply because the accretion disc emission is hidden from us in the X-rays.

disappearance of the continuous jets before the flaring episode starts can be therefore the cause of the flux density dips mentioned above (see Fig.2).

Compact jets are now frequently observed in microquasars in the low-hard X-ray state, although resolved VLBI images are only available for a few of these. The activity cycle of black hole XRB is understood as follows [11]. The X-rays originate in a comptonized corona at the jet base in the hard state. The accretion rate is a few percent Eddington, the jet Lorentz-factor is small $(<2)$. As the accretion rate increases we see an increasing level of hard X-rays and increasing radio emission. The X-rays later get gradually softer and the jet is suppressed (radio quenching). As the system enters to the soft state (at which point most of the X-ray emission is thermal and dominated by the accretion disc), discrete radio ejecta form that are likely to be caused by shocks as either the jet Lorentz factor or the mass flow rate increases. All of this had been observed in SS433 in the radio before this unified picture for black hole XRB was established: the steady compact jets, radio quenching, and the formation of discrete ejecta. However, in case of SS433 the X-ray emission from the accretion is not seen because it is almost completely hidden from us due to absorption and scattering by a dense circumstellar envelope. Another difference is that in SS433 the accretion rate is highly super-Eddington all of the time; still the similarities in the radio behaviour are remarkable. We have to note that the assumption of increasing Lorentz-factor during outbursts is certainly not seen in SS433: the discrete, bright ejecta follow the usual kinematic model with 0.26c. 


\section{The equatorial outflow in SS433 and other black holes: driven by an accretion disk wind?}

Besides the steady inner jets and moving ejecta, there was another type of emission seen in deep radio images, mainly at $1.6 \mathrm{GHz}$. This emitting region surrounds the central source within $\sim 100$ mas: some parts are occasionally detected as distinct features in VLBI images [8], while adding shorter spacings reveals its more diffuse nature [12]. Comparing multi-epoch detections there is evidence for proper motion in this region outwards from the centre, indicating an equatorial outflow [13]. Similar outflows in microquasars and AGN were not known at the time. However, there is now evidence in the X-ray observations for accretion disc driven ultra-fast outflows in both radio-loud [14] and radio-quiet [15] AGN. These may have significant contribution to the AGN feedback. In stellar-mass black holes similar outflows were found in the soft states [16]; these winds may carry sufficient mass and energy to quench the jet radio emission as observed during state transitions. The massive equatorial outflow in SS433 may help us to understand another phenomenon as well. In 2001 it was proposed that X-ray emission in the supercritical accretion disc is channeled towards to the polar regions. When looked at face-on, SS433-like systems may appear as ultra-luminous X-ray sources (ULX) in nearby galaxies [17]. Recently, new evidence was found for highly scattered supercritical disc funnel radiation [18].

\section{Transients and the e-EVN}

During the last 8 years, the real-time e-VLBI developments made the EVN more capable of observing transient radio sources. This is because observations are no longer limited by recording media, the performance of the telescopes can be checked immediately in ad-hoc observations, and the scheduling has become more flexible as well. Responses to flaring activity is now possible in dedicated target of opportunity observations. For example, e-EVN ToO observations of SS433 [19] further confirm that discrete ejecta during flares travel with the same speed as measured in the regular jet, therefore there is no sign of an increasing Lorentz-factor in the jet during accretion disc state transitions. The e-EVN has provided rapid imaging results on several well-known microquasars and other variable or transient sources in recent years. It has proven to be especially useful to combine the e-EVN and VLBA for denser monitoring observations, in which the former can provide initial detections, improved coordinates for new transients, and pre-select nearby secondary calibrator sources for more reliable imaging [e.g. 20]. Most recent results include the identification of a new gamma-ray binary stellar system [21], and the possible localization of the origin of gamma-ray flaring activity in the Crab Nebula [22].

The EVN and MERLIN played an important role in the very beginning of black hole XRB and transient research with the observations of SS433 at the end of 1970s and in the 1980s, and this is the case still today. With the electronic upgrades to e-EVN and e-MERLIN, new opportunities arise: a combination of these two networks will provide a unique range of uv-spacings to image transient sources from mas to arcsecond scales as they expand. In the next few years a great number of new transients will likely be detected by SKA pathfinder instruments and precursors like LOFAR or WSRT APERTIF. The great sensitivity and high resolving power provided by the EVN will be essential to localize and image these at milliarcsecond resolution. 


\section{References}

[1] B. Margon, Observations of SS 433, Ann. Rev. Astron. Astrophys. 22 (1984) 507

[2] R. E. Spencer A radio jet in SS433, Nature 282 (1979) 483

[3] R. T. Schilizzi, C. A. Norman, W. van Breugel, E. Hummel, VLBI detection of SS433, Astron. Astrophys. 79 (1979) 26

[4] I. Fejes, SS 433 extended radio structure observed with the European VLBI Network, Astron. Astrophys. 168 (1986) 69

[5] I. Fejes, Does SS 433 exhibit relativistic beaming?, Astron. Astrophys. 166 (1986) 23

[6] R. C. Vermeulen, Multi-wavelength studies of SS433, PhD thesis, Leiden University, Leiden (1989)

[7] R. C. Vermeulen, R. T. Schilizzi, R. E. Spencer, J. D. Romney, I. Fejes, A series of VLBI images of SS433 during the outbursts in May/June 1987, Astron. Astrophys. 270 (1993) 177

[8] Z. Paragi, R. C. Vermeulen, I. Fejes, R.T. Schilizzi, R. E. Spencer, A. M. Stirling, The inner radio jet region and the complex environment of SS433, Astron. Astrophys. 348 (1999) 910

[9] A. P. Lobanov Ultracompact jets in active galactic nuclei, Astron. Astrophys. 330 (1998) 79

[10] Z. Paragi, R. C. Vermeulen, I. Fejes, R. T. Schilizzi, R. E. Spencer, A. M. Stirling, VLBA multi-frequency monitoring of SS433, New Astron. Rev. 43 (1999) 553

[11] R. P. Fender, T. Belloni, E. Gallo, Towards a unified model for black hole X-ray binary jets, Mon. Not. R. Astron. Soc. 355 (2004) 1105

[12] K. M. Blundell, A. J. Mioduszewski, T. W. B. Muxlow, P. Podsiadlowski, M. P. Rupen, Images of an Equatorial Outflow in SS433, Astrophys. J. 562 (2001) 79

[13] Z. Paragi, I. Fejes, R. C. Vermeulen, R.T. Schilizzi, R. E. Spencer, A. M. Stirling, The Equatorial Outflow of SS433, Proc. EVN Symposium (2002) 263

[14] F. Tombesi, R. M. Sambruna, J. N. Reeves, V. Braito, L. Ballo,J. Gofford, M. Cappi, R. F. Mushotzky, Discovery of Ultra-fast Outflows in a sample of Broad-line Radio Galaxis Observed with Suzaku Astrophys. J. 7192010700

[15] F. Tombesi, M. Cappi, J. N. Reeves, G. G. C. Palumbo, T. Yaqoob, V. Braito, M. Dadina, Evidence for ultra-fast outflows in radio-quiet AGNs I. Detection and statistical incidence of Fe K-shell absorption lines Astron. Astrophys. 521201057

[16] G. Ponti, R. P. Fender, M. C. Begelman, R. J. H., Dunn, J. Neilsen, M. Coriat, Ubiquitous equatorial accretion disc winds in black hole soft states Mon. Not. R. Astron. Soc. 422 (2012) 11

[17] S. Fabrika, A. Mescheryakov, Face-on SS433 stars as a possible new type of extragalactic X-ray sources Proc. IAU Symposium 205 (2001) 268

[18] A. Medvedev, S. Fabrika, Evidence of supercritical disc funnel radiation in X-ray spectra of SS433 Mon. Not. R. Astron. Soc. 402 (2010) 479

[19] V. Tudose, Z. Paragi, S. Thruskin, P. Soleri, R. P. Fender, M. A. Garrett, R. E. Spencer, A. Rushton, P. Burgess, M. Kunert-Bajraszewska, E. Pazderski, K. Borkowski, R. Hammargren, M. Lindqvist, G. Maccaferri, e-VLBI observations of SS433 in outburst Astron. Telegram 1836 (2008) 1

[20] J. Yang, C. Brocksopp, S. Corbel, Z. Paragi, T. Tzioumis, R. P. Fender, A decelerating jet observed by the EVN and VLBA in the X-ray transient XTE J1752-223 Mon. Not. R. Astron. Soc. 409 (2010) 64 
[21] J. Moldón, M. Ribó, J. M. Paredes, Revealing the extended radio emission from the gamma-ray binary HESS J0632+057 Astron. Astrophys. 533 (2011) 7

[22] A. P. Lobanov, D. Horns, T. W. B. Muxlow, VLBI imaging of a flare in the Crab nebula: more than just a spot Astron. Astrophys. 533 (2011) 10 